\title{
Synthesis of Two Four-bar in Series for Body Guidance
}

\author{
Wen-Yeuan Chung \\ Department of Mechanical Engineering, Chinese Culture University, Taipei, Taiwan
}

\begin{abstract}
Using planar two-degree-of-freedom mechanism for the task of body guidance is evaluated and presented. This mechanism consists of two four-bar in series. For some applications of simple planar movement, planar robot with three actuators can be replaced by this mechanism to save cost and reduce complexity. This work reveals that at most seventeen precision positions can be prescribed theoretically. Based on synthetic consideration, each four-bar is proposed to be in charge of some prescribed positions and thus can be designed separately. Decomposing the prescribed precision positions into the assignment of body guidance for each constituent four-bar is developed. Both four-bar are then assembled to successfully execute the task of at most nine precision positions. Numerical example is given to illustrate the synthesis process. Problems of order defect, branch defect and ratio of link lengths are all considered to ensure the performance and applicability of the mechanism.
\end{abstract}

\section{Introduction}

Body guidance is one of major applications for various low-cost linkages. The well-known planar four-bar can be synthesized by prescribing at most five positions theoretically [1]-[2]. The six-bar linkages [3] and a special cam-linkage [4] mechanism were synthesized and introduced for similar tasks. Eleven given positions are expected to be visited by using a complicated eight-bar linkage [5]. The concepts of multi-phase and adjustable link length are also proposed to improve the capability of planar four-bar [6]-[8].

The RRRRR five-link is a two-degree-of-freedom linkage, and the joints at both ends are fixed on the frame and driven by two actuators [9]-[10]. The type of some joints can be prismatic [11]-[12], and the PRRRP parallel mechanism actuated by linear actuators was also proposed [13]. For the purpose of improving performances, such as payload capability and stiffness, the mechanism with seven links including a redundant dyad was suggested [14]-[16]. However, the feature of these linkages is that the middle moving pivot can reach any points within the workspace, and path generation is thus the only application.

An expensive planar robot usually has three DOF (degrees of freedom) and is equipped with three actuators [17]. Hence, its end-effector can be moved to any positions and orientations. The present work focuses on the evaluation and synthesis of mechanisms with two DOF. Body guidance is the major task and the precision positions that can be prescribed should be more than five. Moreover, the synthesized mechanism should have no branch defect and order defect.

\section{Description of mechanism}

Fig. 1 shows the proposed mechanism that mainly consists of two four-bar, $\mathrm{A}_{0} \mathrm{ABB}_{0}$ and $\mathrm{C}_{0} \mathrm{CDD}_{0}$, in series. The driving links are $\mathrm{A}_{0} \mathrm{~A}$ and $\mathrm{C}_{0} \mathrm{C}$. Link CEFD is the guided body. Two frames $X-Y$ and $x_{b}-y_{b}$ are respectively fixed on the base and attached to the guided body. The parameter ${ }^{\mathbf{b}} \mathbf{C}$ represents the coordinate of a point $\mathrm{C}$, being on the guided body, with respect to the frame $x_{b}-y_{b}$. For the guided body CEFD at the $i^{\text {th }}$ prescribed position, the angle between axes $\mathrm{X}$ and $\mathrm{x}_{\mathrm{b}}$ is denoted by $\alpha_{i}$ and the vector between both origins is denoted by $\mathbf{V}_{\mathbf{i}}=\overrightarrow{\mathrm{OO}_{\mathrm{b}}}$. Furthermore, points $\mathrm{A}, \mathrm{B}, \mathrm{C}, \mathrm{C}_{\mathrm{o}}$, $\mathrm{D}$, and $\mathrm{D}_{\mathrm{o}}$ are represented by $\mathrm{A}_{\mathrm{i}}, \mathrm{B}_{\mathrm{i}}, \mathrm{C}_{\mathrm{i}}, \mathrm{C}_{\mathrm{oi}}, \mathrm{D}_{\mathrm{i}}$, and $D_{o i}$ when the guided body is at the $i^{\text {th }}$ prescribed position. The parameter $\mathbf{C}_{\mathbf{i}}$ that is the coordinate of point $\mathrm{C}_{\mathrm{i}}$ with respect to the base frame can thus be derived as

$$
\mathbf{C}_{\mathbf{i}}=\mathbf{R}_{\mathbf{i}}{ }^{\mathrm{b}} \mathbf{C}+\mathbf{V}_{\mathbf{i}}
$$

where

$$
\mathbf{R}_{\mathbf{i}}\left(\alpha_{i}\right)=\left[\begin{array}{cc}
\cos \alpha_{i} & -\sin \alpha_{i} \\
\sin \alpha_{i} & \cos \alpha_{i}
\end{array}\right]
$$

The moving frame $x_{t}-y_{t}$ is attached to the link $\mathrm{AC}_{0} \mathrm{D}_{0} \mathrm{~B}$. Its origin is coincident with point $\mathrm{A}$ and the $x_{t}$ axis is along the vector $\overrightarrow{A B}$. The angle between axes $\mathrm{x}_{\mathrm{t}}$ and $\mathrm{X}$ is denoted by $\beta_{i}$ when the linkage is at the $\mathrm{i}^{\text {th }}$ 
position. Unknown variables $\mathbf{A}_{\mathbf{0}}, \mathbf{B}_{o},{ }^{\mathbf{b}} \mathbf{C},{ }^{\mathbf{b}} \mathbf{D},{ }^{\mathbf{t}} \mathbf{C}_{\mathbf{0}}$, ${ }^{\mathbf{t}} \mathbf{D}_{\mathbf{0}}, \mathbf{x}_{\mathrm{t}}$ component of ${ }^{\mathrm{t}} \mathbf{B}, \mathbf{A}_{\mathbf{i}}$ and $\beta_{i}(\mathrm{i}=1 \sim k+1)$ are assumed, and the total number is $2 \cdot 6+1+(2+1) \cdot(k+1)$. All $\mathbf{D}_{\mathbf{i}}$ can then be derived by using equation like (1). Furthermore, any $\mathbf{B}_{\mathbf{i}}$ can be obtained by

$$
\mathbf{B}_{\mathbf{i}}=\mathbf{R}_{\mathbf{i}}\left(\beta_{i}\right) \cdot{ }^{\mathbf{t}} \mathbf{B}+\mathbf{A}_{\mathbf{i}} \mathrm{i}=1 \sim k+1
$$

All $\mathbf{C}_{\mathbf{o i}}$ and $\mathbf{D}_{\mathbf{o} \mathbf{i}}$ can be derived similarly. After deriving coordinates of all points corresponding to the $i^{\text {th }}$ prescribed position, the conditions that lengths of four links $\mathrm{A}_{\mathrm{o}} \mathrm{A}, \mathrm{B}_{\mathrm{o}} \mathrm{B}, \mathrm{C}_{\mathrm{o}} \mathrm{C}$, and $\mathrm{D}_{\mathrm{o}} \mathrm{D}$ remain constant lead to constraint equations. For example, the equation for the link length $\left|\mathrm{A}_{\mathrm{o}} \mathrm{A}\right|$ is

$$
\begin{gathered}
\left(\mathbf{A}_{\mathbf{o}}-\mathbf{A}_{\mathbf{i}}\right)^{\mathrm{T}} \cdot\left(\mathbf{A}_{\mathbf{o}}-\mathbf{A}_{\mathbf{i}}\right)=\left(\mathbf{A}_{\mathbf{o}}-\mathbf{A}_{\mathbf{1}}\right)^{\mathrm{T}} \cdot\left(\mathbf{A}_{\mathrm{o}}-\mathbf{A}_{\mathbf{1}}\right) \\
\mathrm{i}=2 \sim k+1
\end{gathered}
$$

Therefore, there are $4 \cdot k$ constraints like Eq. 4 . The maximum value of $\mathrm{k}$ can be calculated from the equality

$$
4 \cdot k=2 \cdot 6+1+(2+1) \cdot(k+1)
$$

The value of $\mathrm{k}$ is 16 , and this implies that at most 17 positions can be prescribed by using this mechanism.

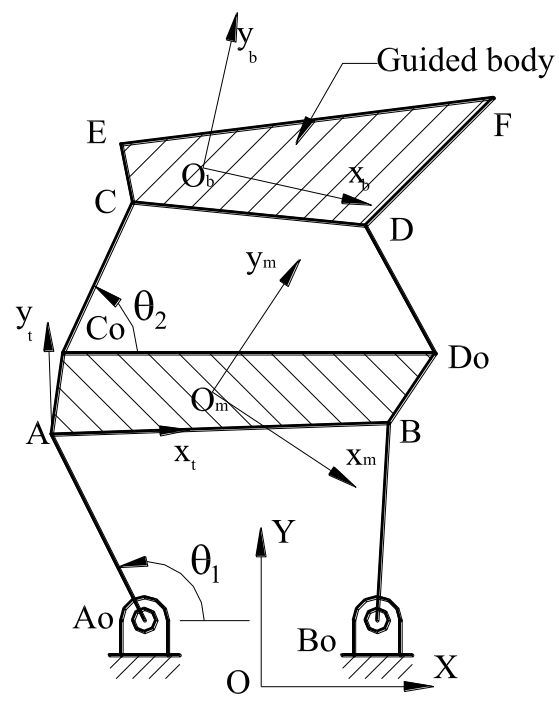

Figure 1. Type III: Seven-link linkage

\section{Synthesis of mechanism}

This mechanism can be synthesized by theoretically solving 16 nonlinear equations after seventeen positions are prescribed, but this task is definitely cumbersome. Even though the solutions can be obtained, the linkage might have order defect and branch defect and is thus useless.

An alternate strategy for applying or synthesizing this mechanism is to let each four-bar be in charge of some prescribed positions separately. For example, if the $\mathrm{j}^{\text {th }}$ prescribed position is under the charge of four-bar $\mathrm{A}_{\mathrm{o}} \mathrm{ABB}_{\mathrm{o}}$, only $\mathrm{A}_{\mathrm{o}} \mathrm{A}$ is actuated when the guided body is moved from the $(\mathrm{j}-1)^{\text {th }}$ position to the $\mathrm{j}^{\text {th }}$ position. Consequently, each four-bar can be synthesized independently as explained in the following.

The coordinates of point $E$ at the $1^{\text {st }}$ and $i^{\text {th }}$ positions can be related by a displacement matrix ${ }_{1}^{\mathrm{i}} \mathbf{T}$ as

$$
\mathbf{E}_{\mathrm{i}}={ }_{1}^{\mathrm{i}} \mathbf{T} \cdot \mathbf{E}_{1}
$$

and

$$
{ }_{1}^{\mathrm{i}} \mathbf{T}=\left[\begin{array}{lc}
\mathbf{R}_{\mathrm{i}} & \mathbf{V}_{\mathrm{i}} \\
\mathbf{0} & 1
\end{array}\right]
$$

Both $\mathbf{E}_{\mathbf{1}}$ and $\mathbf{E}_{\mathbf{i}}$ are 3 by 1 vectors and in the form of $\left[\begin{array}{lll}E_{x} & E_{y} & 1\end{array}\right]^{\mathrm{T}}$ in Eq. 6 and the following derivation. The third moving frame $x_{m}-y_{m}$ is attached to the coupler link $\mathrm{AC}_{\mathrm{o}} \mathrm{D}_{\mathrm{o}} \mathrm{B}$ as shown in Fig. 1. For the $\mathrm{i}^{\text {th }}$ prescribed position, this frame and the fixed $\mathrm{X}-\mathrm{Y}$ frame can be related by a matrix ${ }_{\mathrm{m}}^{\mathbf{f}} \mathbf{T}_{\mathbf{i}}$ and thus

$$
\mathbf{E}_{\mathrm{i}}={ }_{\mathrm{m}}^{\mathrm{f}} \mathbf{T}_{\mathrm{i}} \cdot{ }^{\mathrm{m}} \mathbf{E}_{\mathrm{i}}
$$

The displacement matrix ${ }_{\mathbf{i}}^{\mathbf{j}} \mathbf{N}$ is introduced to describe the movement of four-bar $\mathrm{A}_{\mathrm{o}} \mathrm{ABB}_{\mathrm{o}}$ between the $\mathrm{i}^{\text {th }}$ and $\mathrm{j}^{\text {th }}$ positions. For example, both $\mathbf{B}_{\mathbf{i}}$ and $\mathbf{B}_{\mathbf{j}}$ are related by

$$
\mathbf{B}_{\mathrm{j}}={ }_{\mathrm{i}}^{\mathrm{j}} \mathbf{N} \cdot \mathbf{B}_{\mathrm{i}}
$$

Similarly, the matrix ${ }_{\mathbf{i}}^{\mathrm{j}} \mathbf{M}$ is used to describe the motion of four-bar $\mathrm{C}_{\mathrm{o}} \mathrm{CDD}_{\mathrm{o}}$ with respect to the frame $\mathrm{x}_{\mathrm{m}}-\mathrm{y}_{\mathrm{m}}$. Hence, both ${ }^{\mathrm{m}} \mathbf{E}_{\mathbf{i}}$ and ${ }^{\mathrm{m}} \mathbf{E}_{\mathbf{j}}$ can be related by

$$
{ }^{\mathrm{m}} \mathbf{E}_{\mathrm{j}}={ }_{\mathrm{i}}^{\mathrm{j}} \mathbf{M} \cdot{ }^{\mathrm{m}} \mathbf{E}_{\mathrm{i}}
$$

These displacement matrices, such as ${ }_{\mathbf{i}}^{\mathbf{j}} \mathbf{M},{ }_{\mathbf{i}}^{\mathbf{j}} \mathbf{N}$, or ${ }_{\mathrm{i}}^{\mathrm{j}} \mathbf{T}$, can be multiplied together to obtain a single matrix that relates the first position to the $n^{\text {th }}$ position. For example, ${ }_{1}^{\mathbf{n}} \mathbf{M}$ can be expressed as

$$
{ }_{1}^{n} \mathbf{M}={ }_{n-1}^{n} M \cdot{ }_{n-2}^{n-1} M \ldots{ }_{2}^{3} M \cdot{ }_{1}^{2} M
$$

If all ${ }_{1}^{\mathrm{i}} \mathbf{N}$ and ${ }_{1}^{\mathrm{i}} \mathbf{M}$ can be derived from prescribed motion or ${ }_{1}^{i} \mathbf{T}$, the technique of synthesizing a four-bar as discussed in $[1,2]$ can be followed to synthesize four-bar $\mathrm{A}_{\mathrm{o}} \mathrm{ABB}_{\mathrm{o}}$ and $\mathrm{C}_{\mathrm{o}} \mathrm{CDD}_{\mathrm{o}}$.

Let the movement of the body from the $(\mathrm{j}-1)^{\text {th }}$ position to the $j^{\text {th }}$ position be charged by the four-bar $A_{0} A_{B B}$. The four-bar $\mathrm{C}_{\mathrm{o}} \mathrm{CDD}_{\mathrm{o}}$ thus remains stationary and

$$
{ }_{\mathbf{j}-1}^{\mathrm{j}} \mathbf{M}=\mathbf{I}
$$

The parameter I represents an identity matrix. The matrix ${ }_{j-1}^{j} \mathbf{N}$ is the same as ${ }_{j-1}^{\mathbf{j}-1} \mathbf{T}$ that can be derived from 


$$
{ }_{\mathrm{j}-1}^{\mathrm{j}} \mathbf{T}={ }_{1}^{\mathrm{j}} \mathbf{T} \cdot\left({ }_{1}^{\mathrm{j}-1} \mathbf{T}\right)^{-1}
$$

Both matrixes ${ }_{1}^{\mathrm{j}} \mathbf{N}$ and ${ }_{\mathrm{m}}^{\mathrm{f}} \mathbf{T}_{\mathbf{j}}$ can then be obtained as

$$
\begin{gathered}
{ }_{1}^{j} \mathbf{N}={ }_{j-1}^{j} \mathbf{N} \cdot{ }_{1}^{j-1} \mathbf{N}={ }_{j-1}^{j} \mathbf{T} \cdot{ }_{1}^{j-1} \mathbf{N}={ }_{1}^{j} \mathbf{T} \cdot\left({ }_{1}^{j-1} T\right){ }^{-1} \cdot{ }_{1}^{j-1} \mathbf{N} \\
{ }_{m}^{\mathbf{f}} \mathbf{T}_{j}={ }_{j-1}^{j} \mathbf{N} \cdot{ }_{m}^{\mathrm{f}} \mathbf{T}_{j-1}
\end{gathered}
$$

For the case that the four-bar $\mathrm{C}_{\mathrm{o}} \mathrm{CDD}_{\mathrm{o}}$ is actuated and four-bar $\mathrm{A}_{\mathrm{o}} \mathrm{ABB}_{\mathrm{o}}$ keeps stationary, we have

$$
\begin{aligned}
& { }_{\mathbf{j}-1}^{\mathbf{j}} \mathbf{N}=\mathbf{I} \\
& { }_{\mathbf{m}}^{\mathbf{f}} \mathbf{T}_{\mathbf{j}}={ }_{\mathbf{m}}^{\mathbf{f}} \mathbf{T}_{\mathbf{j}-1}
\end{aligned}
$$

Based on Eq. 6, Eq. 8, and Eq. 10, the term $\mathbf{E}_{\mathbf{j}}$ can be derived respectively as

$$
\begin{aligned}
& \mathbf{E}_{\mathrm{j}}={ }_{1}^{\mathrm{j}} \mathbf{T} \cdot \mathbf{E}_{1}={ }_{1}^{\mathrm{j}} \mathbf{T} \cdot{ }_{\mathrm{m}}^{\mathrm{f}} \mathbf{T}_{1} \cdot{ }^{\mathrm{m}} \mathbf{E}_{1} \\
& \mathbf{E}_{\mathrm{j}}={ }_{\mathrm{m}}^{\mathrm{f}} \mathbf{T}_{\mathrm{j}} \cdot{ }^{\mathrm{m}} \mathbf{E}_{\mathrm{j}}={ }_{\mathrm{m}}^{\mathrm{f}} \mathbf{T}_{\mathrm{j}} \cdot{ }_{1}^{\mathrm{j}} \mathbf{M} \cdot{ }^{\mathrm{m}} \mathbf{E}_{1}
\end{aligned}
$$

By relating both Eq. 18 and Eq. 19, the matrix ${ }_{\mathbf{i}}^{\mathbf{j}} \mathbf{M}$ is derived as

$$
{ }_{1}^{\mathrm{j}} \mathbf{M}=\left({ }_{\mathrm{m}}^{\mathrm{f}} \mathbf{T}_{\mathrm{j}}\right)^{-1} \cdot{ }_{1}^{\mathrm{j}} \mathbf{T} \cdot{ }_{\mathrm{m}}^{\mathrm{f}} \mathbf{T}_{1}
$$

The term ${ }_{\mathrm{m}}^{\mathrm{f}} \mathbf{T}_{1}$ that relates $\mathrm{x}_{\mathrm{m}}-\mathrm{y}_{\mathrm{m}}$ frame and $\mathrm{X}-\mathrm{Y}$ frame for the linkage at the first position is given or can be specified freely. With the responsible four-bar for each prescribed movement being assigned, all matrixes ${ }_{1}^{i} \mathbf{M}$, ${ }_{1}^{\mathrm{i}} \mathbf{N}$, and ${ }_{\mathrm{m}}^{\mathrm{f}} \mathbf{T}_{\mathrm{i}}$ can be derived successively by using Eq. 12, Eq. 14, and Eq. 15 or Eq. 16, Eq. 17, and Eq. 20. Both four-bar are synthesized separately and then assembled with ${ }_{\mathrm{m}}^{\mathrm{f}} \mathbf{T}_{1}$.

\section{Order defect and branch defect}

If a linkage is used to execute body guidance or path generation, problems of branch defect and order defect should be considered and avoided. A four-bar has two configurations for a given input as shown in Fig. 2. If link $\mathrm{A}_{0} \mathrm{~A}$ is the driving, both configurations can be distinguished from the sign of the criterion $\Delta_{i}$.

$$
\Delta_{i}=\left(\left(\mathbf{B}_{\mathbf{i}}-\mathbf{A}_{\mathbf{i}}\right) \times\left(\mathbf{B}_{\mathbf{o}}-\mathbf{B}_{\mathbf{i}}\right)\right) \cdot \hat{\mathbf{k}}
$$

Eq. 21 is a three dimensional vector operation and $\hat{\mathbf{k}}$ is a unit vector normal to the plane. The linkage synthesized should be branch defect-free and associate with the same configuration for all prescribed positions. In other words, the signs of all $\Delta_{i}$ must be the same. On the other hand, the order defect-free implies the satisfaction of the following inequality

$$
\left(\theta_{l j}-\theta_{l i}\right) \cdot\left(\theta_{1 k}-\theta_{l j}\right)>0
$$

where $\theta_{l j}$ represents the value of input angle $\theta_{1}$ corresponding to the $\mathrm{j}^{\text {th }}$ position, and $\mathrm{j}$ is between $\mathrm{i}$ and $\mathrm{k}$.
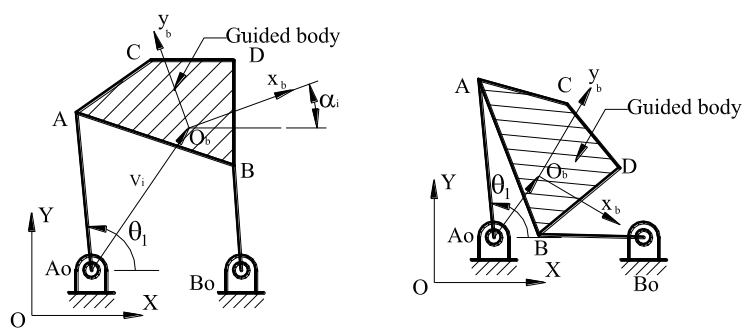

Figure 2. Planar four-bar at two batches

\section{Application and example}

With the assignment of prescribed positions for the mechanism, the results described in Section 3 are used to derive ${ }_{1}^{\mathrm{i}} \mathbf{N}$ and ${ }_{\mathbf{i}}^{\mathrm{j}} \mathbf{M}$ for synthesizing four-bar $\mathrm{A}_{\mathrm{o}} \mathrm{ABB}_{\mathrm{o}}$ and $\mathrm{C}_{\mathrm{o}} \mathrm{CDD}_{\mathrm{o}}$ respectively. The synthetic techniques are inversion, circle point curve, center point curve, and Burmester theory. Any linkages that have order defect or branch defect are discarded based on the criteria described in Section 4.

Example: A mechanism as shown in Fig. 1 is to be designed to execute body guidance. There are eleven prescribed positions as listed in Table 1. Two points $E_{1}:(-2,0)$ and $F_{1}:(-1,0)$ on the coupler link CD are used to verify the movement of body guidance. The precribed movements of points $\mathrm{E}$ and $\mathrm{F}$ or coupler link CD are shown in Fig. 3.

The data of prescribed positions were proposed in [5], and a linkage with eight links and one degree of freedom was synthesized. However, neither order defect nor branch defect was considered.

Table 1. The data of eleven positions

\begin{tabular}{|c|c|c|c|c|c|c|}
\hline & 1 & 2 & 3 & 4 & 5 & 6 \\
\hline$\alpha_{i}$ & 0 & -12.64 & -34.97 & -59.80 & -79.97 & -88.29 \\
\hline$x_{i}$ & 0 & 0.45 & 1.32 & 2.29 & 2.88 & 2.95 \\
\hline$y_{i}$ & 0 & 0.24 & 0.42 & 0.17 & -0.43 & -0.97 \\
\hline & 7 & 8 & 9 & 10 & 11 \\
\hline$\alpha_{i}$ & -79.97 & -59.80 & -34.97 & -12.64 & 0 \\
\hline$x_{i}$ & 2.60 & 1.93 & 1.36 & 1.06 & 1.00 \\
\hline$y_{i}$ & -1.26 & -1.57 & -2.06 & -2.63 & -3.00 \\
\hline
\end{tabular}

Figure 3. Object at eleven prescribed positions 
Sol: 1. By observing the feature of the prescribed positions, the four-bar $\mathrm{A}_{0} \mathrm{ABB}_{0}$ is assigned to guide the link CEFD through the $1^{\text {st }}$ to the $6^{\text {th }}$ positions and four-bar $\mathrm{C}_{\mathrm{o}} \mathrm{CDD}_{\mathrm{o}}$ guides through the other positions. Therefore, each four-bar has to match six prescribed positions

2. Although a four-bar can match precisely at most five prescribed positions based on Burmester theory, the linkages obtained usually have branch defect or even order defect. Therefore, only four of six prescribed positions are chosen to derive the circle point curve, and the others become approximate positions. A linear spaced array covering a specified interval is schemed, and the elements are used for the $\mathrm{x}$ coordinate of pivot $\mathrm{A}_{1}$ or $\mathrm{B}_{1}$. For each $\mathrm{x}$ coordinate, at most three solutions are obtained for pivot $A_{1}$ or $B_{1}$. Numerous four-bar are then synthesized by combining any $A_{1}$ and $B_{1}$. Both branch defect and ratio of link length are checked to discard unacceptable linkages. The others are compared by evaluating the deviations corresponding to both approximate positions. For the $\mathrm{i}^{\text {th }}$ approximate position, the ideal or desired coordinate of pivot A or B can be derived as $\mathbf{A}_{\mathrm{idd}}={ }_{1}^{\mathrm{i}} \mathbf{N} \cdot \mathbf{A}_{\mathbf{1}}$ or $\mathbf{B}_{\mathrm{id}}={ }_{1}^{\mathrm{i}} \mathbf{N} \cdot \mathbf{B}_{\mathbf{1}}$. The deviations are defined by the following functions

$$
\begin{aligned}
& E r r_{i a}=|| A_{o} A_{i d}|-a| / a \\
& E r r_{i b}=|| B_{o} B_{i d}|-b| / b
\end{aligned}
$$

where $a$ is the length of input link $\left|\mathrm{A}_{0} \mathrm{~A}_{1}\right|$ and $b=\left|\mathrm{B}_{0} \mathrm{~B}_{1}\right|$. Four deviations are obtained since there are two approximate positions, and the maximum one is used as the index for comparison. The linkage with the minimum value of index is thus selected. For four-bar $\mathrm{A}_{0} \mathrm{ABB}_{0}$, the $1^{\text {st }}, 4^{\text {th }}, 5^{\text {th }}$, and $6^{\text {th }}$ positions listed in Table 1 are used to derive circle point curve. The data of the best linkage, after comparing the deviations or index, are listed in Table 2.

Table 2. The data of the first four-bar at the first position

\begin{tabular}{|c|c|}
\hline $\mathbf{A}_{\mathbf{o}}$ & $\mathbf{A}_{\mathbf{1}}$ \\
\hline$(1.4373,-1.7443)$ & $(2.0000,1.4667)$ \\
\hline $\mathbf{B}_{1}$ & $\mathbf{B}_{\mathbf{o}}$ \\
\hline$(1.3333,-1.9073)$ & $(6.0457,-4.8063)$ \\
\hline
\end{tabular}

3. The four-bar $\mathrm{C}_{\mathrm{o}} \mathrm{CDD}_{\mathrm{o}}$ is then to be synthesized. Both $\mathrm{x}_{\mathrm{m}}-\mathrm{y}_{\mathrm{m}}$ frame and $\mathrm{X}-\mathrm{Y}$ frame are assumed parallel with translation vector as $(-1.5,2.5)$ when the linkage is at the $1^{\text {st }}$ position, and ${ }_{\mathrm{m}}^{\mathrm{f}} \mathbf{T}_{1}$ is thus defined. The matrix ${ }_{1}^{\mathrm{j}} \mathbf{M}$ is just an identity matrix for $j \leq 6$ since this fourbar remains still. For $j>7$, Eq. 20 has to be used to find ${ }_{1}^{j} \mathbf{M}$. The data like those in Table 1 are obtained and listed in Table 3.

By choosing the $6^{\text {th }}, 7^{\text {th }}, 9^{\text {th }}$, and $11^{\text {th }}$ as the precision positions, this four-bar is synthesized as in Step 2. The coordinates of four pivots with respect to the moving frame $\mathrm{x}_{\mathrm{m}}-\mathrm{y}_{\mathrm{m}}$ are listed in Table 4 .

Table 3. The data of six positions for the second four-bar

\begin{tabular}{|c|c|c|c|c|c|c|}
\hline & 6 & 7 & 8 & 9 & 10 & 11 \\
\hline$\alpha_{i}$ & 0 & 8.32 & 28.49 & 53.32 & 75.65 & 88.29 \\
\hline$x_{i}$ & 0 & -0.066 & -0.442 & -0.359 & 0.309 & 0.927 \\
\hline$y_{i}$ & 0 & -0.602 & -2.056 & -3.831 & -5.272 & -5.934 \\
\hline
\end{tabular}

Table 4. The data of the second four-bar at the first position

\begin{tabular}{|c|c|}
\hline${ }^{\mathrm{m}} \mathbf{C}_{\mathbf{o}}$ & ${ }^{\mathrm{m}} \mathbf{C}_{\mathbf{1}}$ \\
\hline$(3.8714,-2.9097)$ & $(3.3333,-4.9840)$ \\
\hline${ }^{\mathrm{m}} \mathbf{D}_{\mathbf{1}}$ & ${ }^{\mathrm{m}} \mathbf{D}_{\mathbf{~}}$ \\
\hline$(3.3333,-2.5859)$ & $(4.1963,-0.4869)$ \\
\hline
\end{tabular}

4. The motion of each four-bar can be verified by using several animation tools. Both four-bar are then assembled with ${ }_{\mathrm{m}}^{\mathrm{f}} \mathbf{T}_{\mathbf{1}}$ to finish the design. The configurations of the linkage at some positions are shown in Fig. 4. The four-bar $\mathrm{C}_{\mathrm{o}} \mathrm{CDD}_{\mathrm{o}}$ is drawn by dashed lines. The ideal or desired position of segment EF is drawn by bold and solid lines.

5. Fig. 5 shows the stuctural error at eleven prescribed positions. The values are the distances between the actual positions of point $\mathrm{E}$ or $\mathrm{F}$ and the ideal ones. Evidently, the link CEFD can be sequentially guided to the desired positions precisely except for trivial error at the $2^{\text {nd }}, 3^{\text {rd }}$, $8^{\text {th }}$, and $10^{\text {th }}$ positions. The maimum error is less that 0.05 .
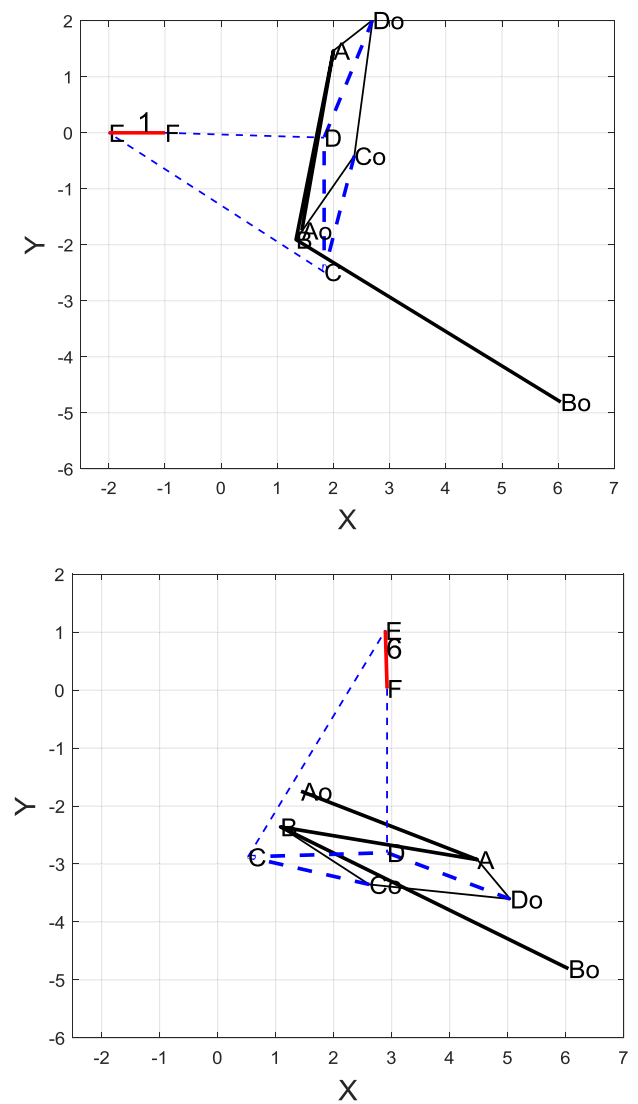


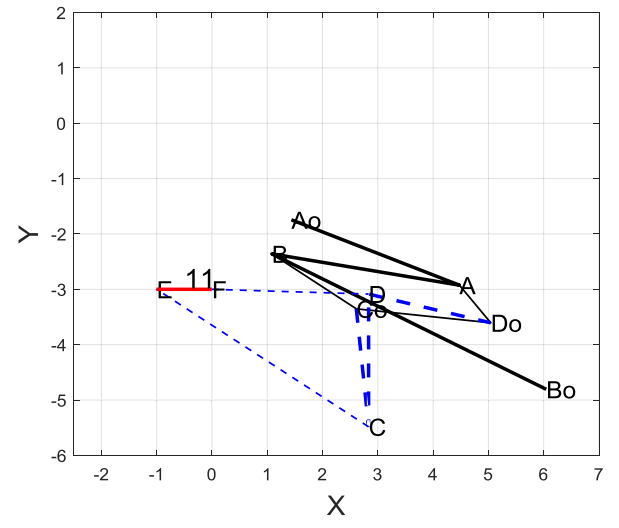

Figure 4. Seven-link mechanism at 1st, 6 th, 11th positions

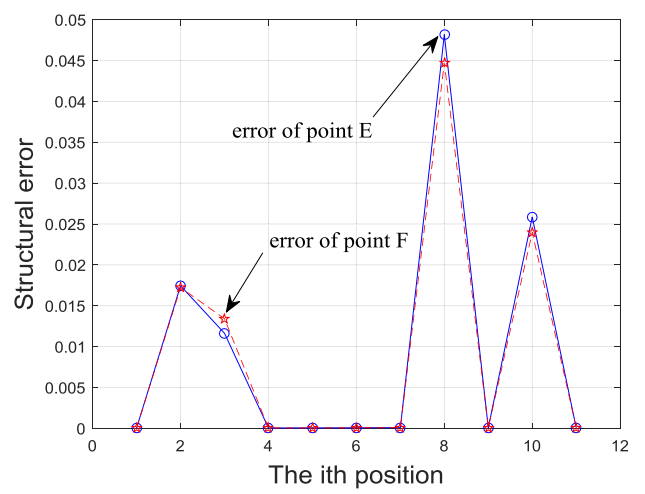

Figure 5. Structural error at eleven positions

\section{Conclusion}

A mechanism with two four-bar in series and with two DOF is proposed for the task of body guidance. Its application and synthesis is presented for the first time. This mechanism can be synthesized by theoretically solving numerous nonlinear equations and at most seventeen positions can be prescribed.

An example illustrates the effective design of this mechanism by mainly actuating each four-bar in sequence. The well-developed techniques for synthesizing four-bar can thus be used. Although the mechanism can guide the body through nine positions precisely, there is few choices left to match other requirements. Therefore, the mechanism synthesized in this example passes through only seven positions precisely. The problems of order defect, branch defect and ratio of link lengths are all considered, and the errors for approximate positions are trivial. The techniques used in this example can certainly be integrated with optimization search so that more positions can be prescribed and more consideration can be included. Evidently, this mechanism can replace complicated and expensive planar robots in some ways.

\section{References}

1. C.S. Suh and C.W. Radcliffe, Kinematics and Mechanisms Design (1978)

2. C.H. Chiang, Kinematics and Design of Planar Mechanisms, Krieger Publishing Company (2000)
3. G.S. Soh and J.M. McCarthy, "The synthesis of sixbar linkages as constrained planar 3R chains," Mechanism and Machine Theory, 45, pp. 160-170 (2008)

4. G. Gatti and D. Mundo, "Optimal synthesis of sixbar cammed-linkages for exact rigid-body guidance," Mechanism and Machine Theory, 42, pp. 1069-1081 (2007)

5. C. Chen and J. Angeles, "A novel family of linkages for advanced motion synthesis," Mechanism and Machine Theory, 43, pp. 882-890 (2008)

6. A. Ahmad and K.J. Waldron, "Synthesis of adjustable planar 4-bar mechanisms", Mechanism and Machine Theory, 14, pp. 405-411 (1979)

7. H.Z. Edmund and H.M. Cheung, "Adjustable fourbar linkages for multi-phase motion generation", Mechanism and Machine Theory, 39, pp. 261-279 (2004)

8. K. Russell and R.S. Sodhi, "On the design of slidercrank mechanisms Part I: multi-phase motion generation", Mechanism and Machine Theory, 40, pp. 285-299 (2005)

9. F. Gao; X.Q. Zhang, Y. S. Zhao, and H. R. Wang, "A physical model of the solution space and the atlas of the reachable workspace for 2-DOF parallel planar manipulators, "Mechanism and Machine Theory, 31, pp. 173-184 (1996)

10. F. Gao, X.Q. Zhang, Y.S. Zhao, and W.B. Zu, "Distribution of some properties in physical model of the solution space of 2-DOF parallel planar manipulators," Mechanism and Machine Theory, 30, pp. 811-817 (1995)

11. J.J. Cervantes-Sánchez and J. G. Rendón-Sánchez, "A simplified approach for obtaining the workspace of a class of 2-dof planar parallel manipulators," Mechanism and Machine Theory, 34, pp. 1057-1073 (1999)

12. T. Huang, M. Li, Z. Li, D.G. Chetwynd, and D.J. Whitehouse, "Optimal kinematic design of 2-DOF parallel manipulators with well-shaped workspace bounded by a specified conditioning index," Transactions on Robotics and Automation, 20, pp. 538-543 (2004)

13. X. J. Liu, J. Wang, and G. Pritschow, "On the optimal kinematic design of the PRRRP 2-DoF parallel mechanism," Mechanism and Machine Theory, 41, pp. 1111-1130 (2006)

14. Y.X. Zhang, S. Cong, W.W. Shang, Z.X. Li, and S.L. Jiang, "Modeling, Identification and Control of a Redundant Planar 2-DOF Parallel Manipulator," International Journal of Control, Automation, and Systems, 5, pp. 559-569 (2007)

15. W.W. Shang, S. Cong, and Y.X. Zhang, "Nonlinear friction compensation of a 2-DOF planar parallel manipulator," Mechatronics, 18, pp. 340-346 (2008)

16. J. Wu, J.S. Wang, and L.P. Wang, "A comparison study of two planar 2-DOF parallel mechanisms: one with 2-RRR and the other with 3-RRR structures," Robotica, 28, pp. 937-942 (2010)

17. J.J. Craig, Introduction to Robotics: Mechanics and Control, Pearson Education (2005) 